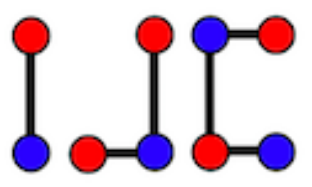

\title{
The degree sequences of a graph with restrictions
}

\author{
Rikio Ichishima ${ }^{\mathrm{a}}$, Francesc A. Muntaner-Batle ${ }^{\mathrm{b}}$, Miquel Rius-Font ${ }^{\mathrm{c}}$, \\ Yukio Takahashi ${ }^{\mathrm{d}}$
}

${ }^{a}$ Department of Sport and Physical Education, Faculty of Physical Education, Kokushikan University,

7-3-1 Nagayama, Tama-shi, Tokyo 206-8515, Japan

${ }^{b}$ Graph Theory and Applications Research Group, School of Electrical Engineering and Computer Science, Faculty of Engineering and Built Environment, The University of Newcastle, NSW 2308 Australia

${ }^{c}$ Departament de Matemàtica Aplicada IV, Universitat Politècnica de Catalunya, C/Esteve Terrades 5, 08860 Castelldefels, Spain

${ }^{d}$ Department of Science and Engineering, Faculty of Electronics and Informations, Kokushikan University, 4-28-1 Setagaya, Setagaya-ku, Tokyo 154-8515, Japan

ichishim@kokushikan.ac.jp, famb1es@yahoo.es, Mriusfont@gmail.com, takayu@kokushikan.ac.jp

\begin{abstract}
Two finite sequences $s_{1}$ and $s_{2}$ of nonnegative integers are called bigraphical if there exists a bipartite graph $G$ with partite sets $V_{1}$ and $V_{2}$ such that $s_{1}$ and $s_{2}$ are the degrees in $G$ of the vertices in $V_{1}$ and $V_{2}$, respectively. In this paper, we introduce the concept of 1-graphical sequences and present a necessary and sufficient condition for a sequence to be 1-graphical in terms of bigraphical sequences.
\end{abstract}

Keywords: degree, degree sequence, graphical sequence, bigraphical sequence, 1-graphical sequence Mathematics Subject Classification: $05 \mathrm{C} 07$

DOI: $10.19184 /$ ijc.2021.5.2.2

\section{Introduction}

We generally follow the notation and terminology pertaining to graphs of [1]. If $F$ is a nonempty subset of the edge set $E(G)$ of a graph $G$, then the subgraph $\langle F\rangle$ induced by $F$ is

Received: 28 July 2020, Revised: 14 September 2021, Accepted: 25 September 2021. 
the graph whose vertex set consists of those vertices of $G$ incident with at least one edge of $F$ and whose edge set is $F$.

The degree of a vertex $v$ in a graph $G$ is the number of edges of $G$ incident with $v$, which is denoted by $\operatorname{deg} v$. A vertex is called even or odd according to whether its degree is even or odd.

A sequence $d_{1}, d_{2}, \ldots, d_{n}$ of nonnegative integers is called a degree sequence of a graph $G$ if the vertices of $G$ can be labeled $v_{1}, v_{2}, \ldots, v_{n}$ so that $\operatorname{deg} v_{i}=d_{i}$ for all $i$. We adopt the convention that the vertices have been labeled so that $d_{1} \geq d_{2} \geq \cdots \geq d_{n}$. We call a sequence of nonnegative integers graphical if it is the degree sequence of some graph. A necessary and sufficient condition for a sequence to be graphical was found by Havel [4] and later rediscovered by Hakimi [3].

Theorem 1.1. A sequence $s: d_{1}, d_{2}, \ldots, d_{n}$ of nonnegative integers with $d_{1} \geq d_{2} \geq \cdots \geq d_{n}, n \geq$ $2, d_{1} \geq 1$ is graphical if and only if the sequence $s^{\prime}: d_{2}-1, d_{3}-1, \ldots, d_{d_{1}+1}-1, d_{d_{1}+2}, \ldots, d_{n}$ is graphical.

According to the definition of a simple graph, two distinct vertices are joined by at most one edge. If we allow more than one edge (but a finite number) to join pairs of vertices, the resulting structure is called a multigraph. If two or more edges join the same two vertices in a multigraph, then these edges are referred to as multiple edges. Hakimi [3] extended the preceding result to multigraphs.

Theorem 1.2. Let $d_{1}, d_{2}, \ldots, d_{n}$ be nonnegative integers with $d_{1} \geq d_{2} \geq \cdots \geq d_{n}$ and $n \geq 2$. Then there exists a multigraph with degree sequence $s: d_{1}, d_{2}, \ldots, d_{n}$ if and only if $\sum_{i=1}^{n} d_{i}$ is even and $d_{1} \leq d_{2}+d_{3}+\cdots+d_{n}$.

Two finite sequences $s_{1}$ and $s_{2}$ of nonnegative integers are called bigraphical if there exists a bipartite graph $G$ with partite sets $V_{1}$ and $V_{2}$ such that $s_{1}$ and $s_{2}$ are the degrees in $G$ of the vertices in $V_{1}$ and $V_{2}$, respectively. The following result is an analog of Theorem 1.1 for graphs (see [1, p. 16]).

Theorem 1.3. The sequences $s_{1}: a_{1}, a_{2}, \ldots, a_{r}$ and $s_{2}: b_{1}, b_{2}, \ldots, b_{t}$ of nonnegative integers with $r \geq 2, a_{1} \geq a_{2} \geq \cdots \geq a_{r}, b_{1} \geq b_{2} \geq \cdots \geq b_{t}, 0<a_{1} \leq t, 0<b_{1} \leq r$ are bigraphical if and only if $s_{1}^{\prime}: a_{2}, a_{3}, \ldots, a_{r}$ and $s_{2}^{\prime}: b_{1}-1, b_{2}-1, \ldots, b_{a_{1}}-1, b_{a_{1}+1}-1, \ldots, b_{t}$ are bigraphical.

The outdegree od $v$ of a vertex $v$ of a digraph $D$ is the number of vertices of $D$ that are adjacent from $v$, while the indegree id $v$ of $v$ is the number of vertices of $D$ adjacent to $v$. The degree $\operatorname{deg} v$ of a vertex $v$ of $D$ is defined by

$$
\operatorname{deg} v=\operatorname{od} v+\operatorname{id} v .
$$

A loop is an edge that joins a vertex to itself and contributes to the degree of a vertex twice. A graph $G$ is called a 1-graph if it has at most one loop attached at each vertex and at most two multiple edges joining each pair of vertices. A sequence $s$ is called 1-graphical if there exists a 1-graph that realizes $s$.

For the sake of notational convenience, we will denote the interval of integers $x$ such that $a \leq x \leq b$ by simply writing $[a, b]$.

In this paper, we present a necessary and sufficient condition for a sequence to be 1-graphical in terms of bigraphical sequences. To this end, we use the following theorem, due to Veblen [7], which characterizes eulerian graphs in terms of their cycle structures. 
Theorem 1.4. A nontrivial connected graph $G$ is eulerian if and only if $E(G)$ can be partitioned into subsets $E_{i}, i \in[1, k]$, where each subgraph $\left\langle E_{i}\right\rangle$ is a cycle.

To conclude this introduction, it is worth to mention that López and Muntaner-Batle [5] completely characterized the degree sequences of graphs with at most one loop attached at each vertex and no multiple edges. Hence, the work conducted in this paper would be a natural continuation of their work.

\section{Characterization of 1-graphical sequences}

We are now ready to state and prove the following theorem.

Theorem 2.1. A sequence $s: d_{1}, d_{2}, \ldots, d_{n}$ of nonnegative integers with $d_{1} \geq d_{2} \geq \cdots \geq d_{n}$ and $n \geq 2$ is 1-graphical if and only if there exist bigraphical sequences $s_{1}: a_{1}, a_{2}, \ldots, a_{n}$ and $s_{2}: b_{1}, b_{2}, \ldots, b_{n}$ such that $a_{i}=b_{i}=d_{i} / 2$ for even $d_{i}$ and $a_{i}+b_{i}=d_{i}$ for odd $d_{i}$, where $i \in[1, n]$.

Proof. By assumption, there exists a 1-graph that realizes $s$. Assume that the vertices of $G$ are labeled $v_{1}, v_{2}, \ldots, v_{n}$ so that $\operatorname{deg} v_{i}=d_{i}$ for all $i$, and construct a new graph $H$ with vertex set

$$
V(H)=V(G) \cup\{u\} \quad \text { and edge set } \quad E(H)=E(G) \cup\left\{u v_{i} \mid d_{i} \text { is odd }\right\} .
$$

Since in any graph, there is an even number of odd vertices, it follows that all vertices in $H$ are even vertices. Therefore, since every component of $H$ is eulerian, it follows from Theorem 1.4 that $E(H)$ can be partitioned into subsets $E_{i}, i \in[1, k]$, where each subgraph $\left\langle E_{i}\right\rangle$ is a cycle. If we orient each cycle in $\left\langle E_{i}\right\rangle$ cyclically, then we obtain a digraph $D$ with the property that od $v=$ id $v$ for every $v \in V(D)$. Now, let $D^{\prime}$ be the digraph obtained by deleting the vertex $u$ from $D$. Certainly, $d_{i}=$ od $v+$ id $v$ for each $v \in V\left(D^{\prime}\right)$. Furthermore, the vertices of $D^{\prime}$ have the properties that od $v=\mathrm{id} v=d_{i} / 2$ for even $d_{i}$ and $|\operatorname{od} v-\mathrm{id} v|=1$ for odd $d_{i}$, where $i \in[1, n]$. Let $A\left(D^{\prime}\right)=\left[\alpha_{i j}\right]$ be the adjacency matrix of $D^{\prime}$, and construct the bipartite digraph $D^{*}$ with partite sets

$$
X=\left\{x_{i} \mid 1 \leq i \leq n\right\} \text { and } Y=\left\{y_{i} \mid 1 \leq i \leq n\right\},
$$

and with the arcs in such a way that $\left(x_{i}, y_{j}\right) \in E\left(D^{*}\right)$ if and only if $\alpha_{i j}=1$. It remains to observe that the sequences of outdegrees and of indegrees satisfy the required properties.

Let $G$ be the bipartite graph with partite sets

$$
X=\left\{x_{i} \mid i \in[1, n]\right\} \text { and } Y=\left\{y_{i} \mid i \in[1, n]\right\}
$$

such that $s_{1}$ and $s_{2}$ are the degrees in $G$ of the vertices in $X$ and $Y$, respectively. Further, consider the digraph $D$ obtained from $G$, and let $\left[\beta_{i j}\right]$ be the $n \times n$ matrix with $\beta_{i j}=1$ if and only if $\left(x_{i}, y_{j}\right)$ is an arc of $D$ and $\beta_{i j}=0$ otherwise. Let $D^{\prime}$ be the digraph with the vertex set $V\left(D^{\prime}\right)=$ $\left\{w_{i} \mid 1 \leq i \leq n\right\}$ and adjacency matrix $\left[\beta_{i j}\right]$ so that od $w_{i}+\mathrm{id} w_{i}=a_{i}+b_{i}=d_{i}$. Then the graph obtained by replacing each arc $(u, v)$ of $D^{\prime}$ by the edge of $u v$ is a 1-graph that realizes a sequence $s$.

This result has the following consequences. 
The degree sequences of ...

Corollary 2.1. Let $s: d_{1}, d_{2}, \ldots, d_{n}$ be a sequence of nonnegative even integers with $d_{1} \geq d_{2} \geq$ $\cdots \geq d_{n}$ and $n \geq 2$. Then $s$ is 1-graphical if and only if the sequences

$$
s_{1}=s_{2}: \frac{d_{1}}{2}, \frac{d_{2}}{2}, \ldots, \frac{d_{n}}{2}
$$

are bigraphical.

Corollary 2.2. Let $s: d_{1}, d_{2}, \ldots, d_{n}$ be a sequence of nonnegative integers with $d_{1} \geq d_{2} \geq$ $\cdots \geq d_{n}, n \geq 2$ and the properties that $d_{i}=d_{i+1}$ for $k \leq i \leq k+l-1, d_{i}$ is even for all $i \in[1, k-1] \cup[k+l+1, n]$ and $d_{i}$ is odd for all $i \in[1, k+l]$. Then $s$ is 1-graphical if and only if the sequences

$$
\begin{array}{r}
s_{1}=s_{2}: \frac{d_{1}}{2}, \frac{d_{2}}{2}, \ldots, \frac{d_{k-1}}{2},\left\lceil\frac{d_{k}}{2}\right\rceil,\left\lceil\frac{d_{k+1}}{2}\right\rceil, \ldots,\left\lceil\frac{d_{k+(l-1) / 2}}{2}\right\rceil, \\
\left\lfloor\frac{d_{k+(l+1) / 2}}{2}\right\rfloor, \ldots,\left\lfloor\frac{d_{k+l}}{2}\right\rfloor, \ldots, \frac{d_{k+l+1}}{2}, \frac{d_{k+l+2}}{2}, \ldots, \frac{d_{n}}{2}
\end{array}
$$

are bigraphical.

Corollary 2.3. Let $s: d_{1}, d_{2}, \ldots, d_{n}$ be a sequence of nonnegative integers with $d_{1} \geq d_{2} \geq \cdots \geq$ $d_{n}, n \geq 2$ and the properties that there exist some integers $k$ and $l, 1 \leq k<l \leq n$, so that $d_{k}$ and $d_{l}$ are odd, and $d_{i}$ is even for all $i \in[1, n] \backslash\{k, l\}$. Then $s$ is 1-graphical if and only if the sequences

$$
s_{1}: \frac{d_{1}}{2}, \frac{d_{2}}{2}, \ldots, \frac{d_{k-1}}{2},\left\lceil\frac{d_{k}}{2}\right\rceil, \frac{d_{k+1}}{2}, \ldots, \frac{d_{l-1}}{2},\left\lfloor\frac{d_{l}}{2}\right\rfloor, \frac{d_{l+1}}{2}, \ldots, \frac{d_{n}}{2}
$$

and

$$
s_{2}: \frac{d_{1}}{2}, \frac{d_{2}}{2}, \ldots, \frac{d_{k-1}}{2},\left\lfloor\frac{d_{k}}{2}\right\rfloor, \frac{d_{k+1}}{2}, \ldots, \frac{d_{l-1}}{2},\left\lceil\frac{d_{l}}{2}\right\rceil, \frac{d_{l+1}}{2}, \ldots, \frac{d_{n}}{2}
$$

are bigraphical.

\section{Conclusions}

The bipartite realization problem formulates as follows: Given two finite sequences $s_{1}$ and $s_{2}$ of nonnegative integers, is there a labeled bipartite graph such that the pair of $s_{1}$ and $s_{2}$ is the degree sequence of some bipartite graph? This classical decision problem belongs to the complexity class of P. This can be proven by two known approaches established in 1957 by Gale [2] and also by Ryser [6]. In this paper, we have extended Theorem 1.2 by presenting necessary and sufficient conditions for a sequence of nonnegative integers to be 1-graphical in terms of bigraphical sequences. These together with the bipartite realization problem imply that the decision problem associated with determining whether a given sequence of nonnegative integers is 1-graphical remains to be the complexity class of $\mathrm{P}$.

\section{Acknowledgement}

The second and third authors would like to acknowledge Dr. Keith Edwards for the discussions maintained with us during the elaboration of this work. 


\section{References}

[1] G. Chartrand and L. Lesniak, Graphs \& Digraphs 3th ed. CRC Press (1996).

[2] D. Gale, A theorem on flows in networks, Pacific J. Math. 7 (2) (1957), 1073-1082.

[3] S. Hakimi, On the realizability of a set of integers as degrees of the vertices of a graph, $J$. SIAM Appl. Math. 10 (1962), 496-506.

[4] V. Havel, A remark on the existence of finite graphs (Czech), Časopis Pĕst. Math. 80 (1955), 477-480.

[5] S. C. López and F. A Muntaner-Batle, Mirror bipartite graphs, preprint, 2013. Available at https://ui.adsabs.harvard.edu/abs/2013arXiv1305.5145L/abstract.

[6] H. J. Ryser, Combinatorial properties of matrices of zeros and ones, Can. J. Math. 9 (1957), 371-377.

[7] O. Veblen, An application of modular equations in analysis situs, Math. Ann. 14 (1912-1913), 86-94. 